\title{
Immunogenicity of Three Different Influenza Vaccines against Homologous and Heterologous Strains in Nursing Home Elderly Residents
}

\author{
Vincenzo Baldo, ${ }^{1}$ Tatjana Baldovin, ${ }^{1}$ Michele Pellegrini, ${ }^{2}$ Gabriele Angiolelli, ${ }^{3}$ \\ Silvia Majori, ${ }^{4}$ Annarosa Floreani, ${ }^{5}$ Marta Cecilia Busana, ${ }^{1}$ Chiara Bertoncello, ${ }^{1}$ \\ and Renzo Trivello ${ }^{1}$ \\ ${ }^{1}$ Department of Environmental Medicine and Public Health, Institute of Hygiene, University of Padua, Via loredan 18, \\ 35151 Padua, Italy \\ ${ }^{2}$ Global Clinical Research and Development, Novartis Vaccines \& Diagnostics S.r.l., Via Fiorentina 1, 53100 Siena, Italy \\ ${ }^{3}$ Local Health Unit n.13, Veneto Region, Via L. Mariutto, 76-30035 Mirano, Italy \\ ${ }^{4}$ Department of Medicine and Public Health, Section of Hygiene and Preventive, Environmental and Occupational Medicine, \\ University of Verona, Strada Le Grazie, 8-37134 Verona, Italy \\ ${ }^{5}$ Department of Surgical and Gastroenterological Sciences, University of Padua, Via Giustiniani 2, 35100 Padua, Italy
}

Correspondence should be addressed to Vincenzo Baldo, vincenzo.baldo@unipd.it

Received 25 August 2009; Accepted 7 January 2010

Academic Editor: Jiri Mestecky

Copyright (C) 2010 Vincenzo Baldo et al. This is an open access article distributed under the Creative Commons Attribution License, which permits unrestricted use, distribution, and reproduction in any medium, provided the original work is properly cited.

\begin{abstract}
We studied whether MF59-adjuvanted influenza vaccine improves immunity against drifted influenza strains in institutionalised elderly with underling chronic health conditions. Sera from a randomized study, comparing MF59-adjuvanted (Sub/MF59, $n=72)$, virosomal (SVV, $n=39)$, and split $(n=88)$ vaccines, were retested using a hemagglutination inhibition (HI) assay against homologous (Northern Hemisphere [NH] 1998/99) and drifted (NH 2006/07) strains. Corrected postvaccination HI antibody titres were significantly higher with Sub/MF59 than SVV for all strains; GMTs against homologous A/H3N2 and B and both drifted A strains were significantly higher for Sub/MF59 than split. Seroprotection rates and mean-fold titer increases were generally higher with Sub/MF59 for all A influenza strains. MF59-adjuvanted influenza vaccine induced greater and broader immune responses in elderly people with chronic conditions, than conventional virosomal and split vaccines, particularly for $\mathrm{A} / \mathrm{H} 1$ and $\mathrm{A} / \mathrm{H} 3$ strains, potentially giving clinical benefit in seasons where antigenic mismatch occurs.
\end{abstract}

\section{Introduction}

The frequency and severity of infectious diseases, including influenza, increase with old age. The elderly are particularly vulnerable to influenza and this highly contagious infectious disease causes a high frequency of morbidity and mortality in older individuals [1-4]. The mortality rate in the elderly is particularly high compared with the general population, with $95 \%$ of all influenza-related deaths occurring in the elderly, primarily in those with underlying chronic health conditions [5].
Groups at high-risk of complications of influenza include patients with cardiovascular or pulmonary conditions, metabolic diseases, and the institutionalized [6]. In fact, influenza can exacerbate underlying diseases in the elderly population, being the likely primary cause of the winterseason increase in mortality in patients with ischemic heart disease, cerebrovascular disease and diabetes mellitus $[7,8]$.

Annual vaccination is the recommended method to prevent influenza; the WHO has suggested that vaccination can reduce influenza-related morbidity by $60 \%$ and 
influenza-related mortality by $70-80 \%$ [9]. However, currently available influenza vaccines have demonstrated limited efficacy in the elderly, mainly because of the waning immune response typical with advancing age [10-12]. Indeed, lower IgA and IgG antibody responses, delayed peak antibody titers, and a faster decline in titers following vaccination are observed, especially in very old and frail persons [13].

The continual evolution of the influenza virus also impacts on the effectiveness of influenza vaccines. Antigenic drift frequently occurs in influenza A and B subtypes and the impact of this drift on vaccine effectiveness in the elderly is considered very high [14-16]. It has been suggested that antigenic drift is associated with a more severe and early onset of influenza epidemic, since the level of preexisting immunity to the drifted strain is reduced [17]. In elderly subjects seroprotection rates can be as low as $20 \%$ against drifted strains, dropping from $\geq 70 \%$ in years where a good antigenic match is observed [18-21].

Meeting the challenge presented by waning immunity in the elderly requires vaccines that offer enhanced immunogenicity and increased clinical protection, such as adjuvanted influenza vaccines. Formulation of a subunit influenza vaccine with the MF59 adjuvant has been shown to enhance immunogenicity and offer broader serological protection in the elderly compared with conventional non-adjuvanted vaccines, especially versus the most epidemiologically prevalent $\mathrm{A} / \mathrm{H} 3 \mathrm{~N} 2$ influenza viruses $[6,20,22]$.

This study was performed to assess the immunogenicity of three inactivated influenza vaccines, a MF59-adjuvanted subunit vaccine (Sub/MF59; FLUAD ${ }^{\circledR}$, Novartis Vaccines), a virosomal vaccine $\left(\mathrm{SVV}\right.$; InflexalV ${ }^{\circledR}$, Swiss Serum and Vaccine Institute), and a split vaccine (Split; Mutagrip ${ }^{\circledR}$, Pasteur Merieux MSD), against homologous and heterologous strains, by retesting sera of elderly nursing home residents with chronic underlying conditions, who participated in a previous randomized, controlled trial [6].

\section{Materials and Methods}

Sera from a subset of 199 elderly nursing home residents ( $\geq 65$ years of age) previously enrolled in a randomized, controlled trial performed during the winter season of 1998/99 [6], were reanalyzed to test the immunogenicity conferred by MF59-adjuvanted influenza vaccine (Sub/MF59; $n=72$ ), by a virosomal (SVV, $n=39$ ) and a split (Split; $n=88$ ) vaccines against homologous and heterologous influenza strains.

During the clinical study, after obtaining informed consent, blood samples (approximately $10 \mathrm{~mL}$ ) were drawn prior to and 4 weeks after vaccination. Sera were stored at $-20^{\circ} \mathrm{C}$ until laboratory determination of $\mathrm{HI}$ antibody titres, as described elsewhere [23].

All subjects received a single $0.5 \mathrm{~mL}$ intramuscular (IM) dose of Sub/MF59, virosomal or split vaccine in the deltoid region of the non-dominant arm. Each vaccine dose contained $15 \mu \mathrm{g}$ of hemagglutinin of each of the influenza strains recommended by the WHO for the 1998/99 Northern Hemisphere (NH) influenza season: A/Sydney/5/97(A/H3N2)-like virus, A/Beijing/262/95(A/H1N1)-like virus and B/Beijing/ 184/93-like virus.

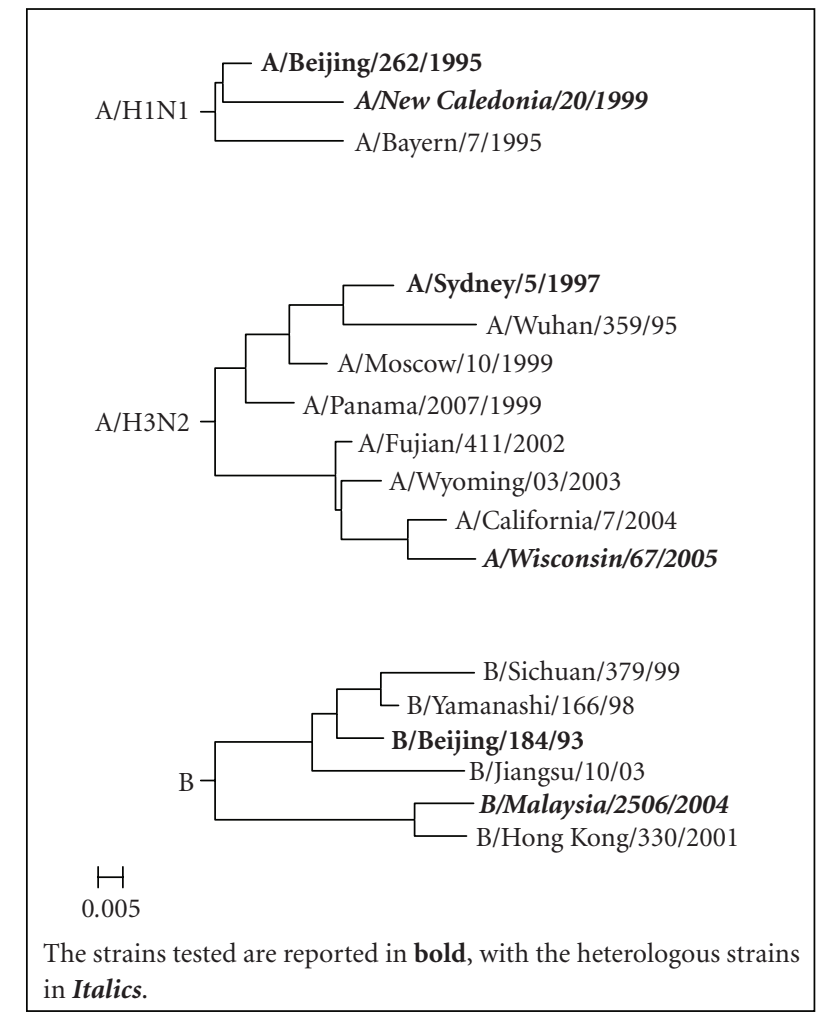

FIGURE 1: Hemagglutinin phylogenetic trees of the strains recommended for NH vaccine formulation from 1997/1998 to 2006/2007 influenza seasons.

The study was conducted in compliance with the Italian Law Decree on the protection of personal data.

2.1. Assessment of Vaccine Immunogenicity. The current analysis tested the immunogenicity using the $\mathrm{HI}$ assay against the homologous 1998/99 strains and the heterologous viral strains recommended for the 2006/07 NH influenza season [A/Wisconsin/67/2005 (A/H3N2)-like virus; A/New Caledonia/20/99 (A/H1N1)-like virus and B/Malaysia/2506/2004like virus]. The heterologous $B$ strain selected for immunogenicity analyses belongs to the $\mathrm{B} /$ Victoria/2/87 lineage, whereas the $\mathrm{B}$ strain included in the vaccine formulation (B/Beijing) was from the alternative phylogenetic line of B/Yamagata/16/88, with a previously reported lack of crossreactivity between antibodies for these two divergent lineages [24].

Phylogenetic trees of the influenza strains analyzed in the study (both homologous and heterologous), including the vaccine strains recommended for $\mathrm{NH}$ formulation from $1997 / 98$ to 2006/07 influenza seasons, was based on sequence analysis of the region codifying for the hemagglutinin (Figure 1). Data on influenza strains was obtained from the website of the National Institute of Allergy and Infectious Diseases-funded Influenza Research Database (NIAID IRD; http://www.fludb.org), and phylogenetic analyses were conducted using the software Molecular Evolutionary Genetics Analysis (MEGA), version 4.0 [25]. 
The study parameters considered as expression of humoral immune response were: the Geometric Mean Titers (GMTs) of HI antibodies with $95 \%$ confidence intervals (CI); post-vaccination mean-fold increase (MFI) in HI antibodies (ratio of post- to pre-vaccination titres); the number of subjects with protective HI antibody level (titre $\geq 40$ was considered protective); and the number of subjects with at least a 4 -fold increase in post-vaccination titres.

2.2. Statistical Analysis. Data were analyzed using the Statistical Package for the Social Sciences (SPSS, Chicago, IL, USA). The chi-square test was performed to analyze differences between proportions of subjects. Statistical significance between pre- and postvaccination titres was calculated using the paired Student's $t$-test. Comparison of different vaccine groups was determined by Student's $t$-test for unpaired data.

A multivariate logistic regression analysis was carried out for each virus strain to determine variables that are independently associated with the likelihood of a person achieving at least a 4 -fold increase in $\mathrm{HI}$ titres. A $P$-value of $<.05$ was considered to indicate statistical significance, with an odds ratio $(\mathrm{OR})$ of $<1$ signifying that a person was less likely to achieve a 4 -fold increase.

Correction of the GMTs for pre-vaccination status was performed: absolute titres were divided by 5 (i.e., undetectable titre $<10)$ and then transformed to binary logarithms. On this scale, an undetectable (seronegative) titre represents "0", a titre of 10 represents "1", a titre of 20 represents " 2 ", and so forth, HI titres were transformed into binary logarithms and corrected for pre-vaccination status as described by Beyer et al. [26].

\section{Results}

Sera were available from 199 elderly who took part into the original study, and were retested for these new immunogenicity analyses for the Sub/MF59 $(n=72)$, SVV $(n=39)$ and Split $(n=88)$ groups. According to the original baseline characteristics more healthy subjects populated the split group, compared with Sub/MF59 and SVV. The majority of subjects in these last two groups $(87.5 \%$ and $79.5 \%$, resp.) had at least one underlying chronic condition, including cardiac and pulmonary conditions, or diabetes mellitus. More than $80 \%$ of subjects in each group were $>75$ years of age. The demographic characteristics of the subjects, recorded at time of the original study, are summarized in Table 1.

No statistically significant differences were found in terms of baseline GMTs between vaccine groups versus homologous or heterologous strains.

The antibody responses are shown in Table 2 and Figure 2, according to viral strain and vaccine group. Vaccination elicited higher GMTs against all strains, in all three vaccination groups (Figure 2).

3.1. Immunogenicity against Homologous Strains. Very high seroprotection rates (SP) were achieved by all three vaccines (Table 2), but vaccination with Sub/MF59 resulted in slightly but consistently higher postvaccination GMTs than the other
TABLE 1: Baseline characteristics of the study groups.

\begin{tabular}{lccc}
\hline Subgroup & $\begin{array}{c}\text { Sub/MF59 } \\
(n=72) \\
n(\%)\end{array}$ & $\begin{array}{c}\text { Split }(n=88) \\
\text { SVV }(n=39)\end{array}$ & $n(\%)$ \\
\hline $\begin{array}{l}\text { Age group, years } \\
\quad \leq 85\end{array}$ & $31(43.1)$ & $42(47.7)$ & $11(28.2)$ \\
$\quad>85$ & $41(56.9)$ & $46(52.3)$ & $28(71.8)$ \\
Gender & & & \\
$\quad$ Males & $5(6.9)$ & $24(27.3)$ & $0(0.0)$ \\
$\quad$ Females & $67(93.1)$ & $64(72.7)$ & $39(100)$ \\
Previously vaccinated & & & \\
$\quad$ No & $12(16.7)$ & $16(18.2)$ & $2(5.1)$ \\
$\quad$ Yes & $60(83.3)$ & $76(81.8)$ & $37(94.9)$ \\
Underlying disease & & & \\
$\quad$ No & $9(12.5)$ & $35(39.8)$ & $8(20.5)$ \\
$\quad$ Yes* & $63(87.5)$ & $53(60.2)$ & $31(79.5)$ \\
Heart condition & $51(70.8)$ & $49(55.7)$ & $24(61.5)$ \\
Lung condition & $42(58.3)$ & $11(12.5)$ & $17(43.6)$ \\
Diabetes mellitus & $12(16.7)$ & $8(9.1)$ & $3(7.7)$ \\
Other & $14(19.4)$ & $2(2.3)$ & $9(23.1)$ \\
\hline
\end{tabular}

* More than one risk status was possible for each subject.

two vaccination groups (Figure 2). In particular, Sub/MF59 induced statistically significantly higher HI antibody titers for all three strains, than those of the SVV vaccine group. The GMTs were substantially confirmed after correction for baseline titers (Figure 3), and Sub/MF59 vaccine showed the ability to induce statistically significant $(P<.01)$ higher GMTs against $\mathrm{A} / \mathrm{H} 3$ and $\mathrm{B}$ homologous strains, compared to the split vaccine.

Postvaccination mean-fold increases in HI antibodies were greater with Sub/MF59 compared with both virosomal and split vaccines (Table 2).

Significantly more subjects achieved at least a 4 -fold increase in $\mathrm{HI}$ titres against the homologous B strain following Sub/MF59 vaccination, than the virosomal vaccine (66.7\% versus $46.2 \%$, resp., $P=.03$ ) (Table 2 ).

3.2. Immunogenicity against Heterologous Strains. Consistently higher GMTs were reported in the Sub/MF59 group, and these values were statistically significant for the A/H3N2 and $\mathrm{A} / \mathrm{H} 1 \mathrm{~N} 1$ strains (Figure 2), compared with both nonadjuvanted vaccines. After Beyer's correction, the HI antibody titers against $B$ strains were significantly higher in the Sub/MF59 than in the virosomal group.

Post-vaccination mean-fold increases in $\mathrm{HI}$ antibodies were greater with Sub/MF59 compared with the other two vaccine groups against all the three influenza virus variants (Table 2).

Seroprotection rates induced by Sub/MF59 group were higher than those after the SVV injection for both A strains $(P<.01$ versus $\mathrm{H} 1 \mathrm{~N} 1, P<.05$ versus A/H3N2). Similar SP rates against the $B$ drifted strain were found in the three vaccine groups. 


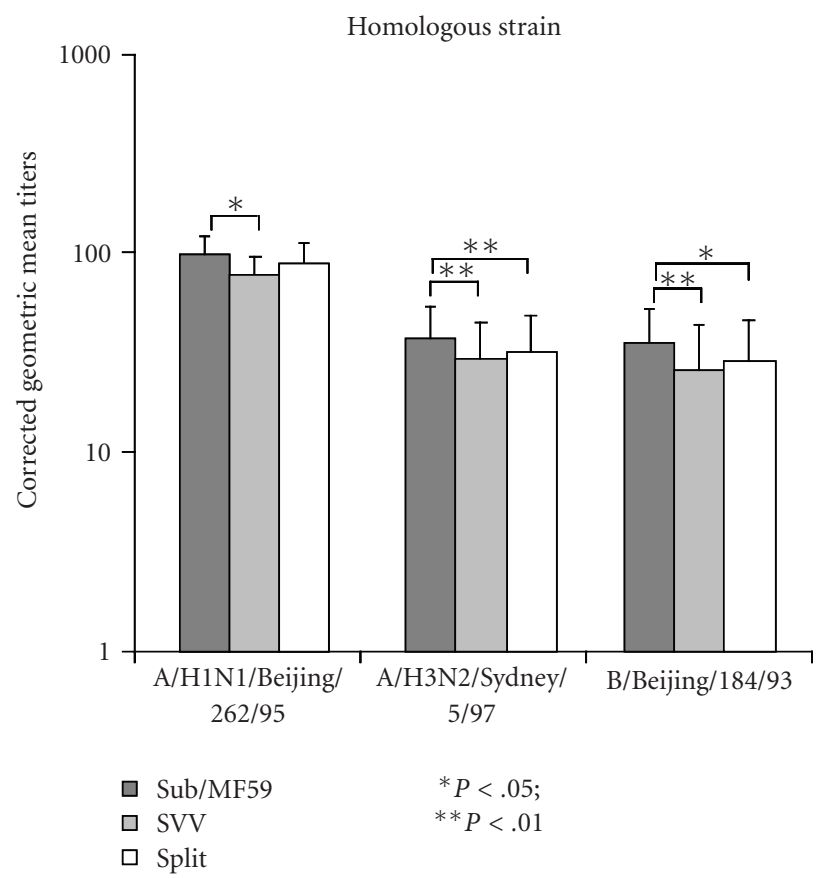

(a)

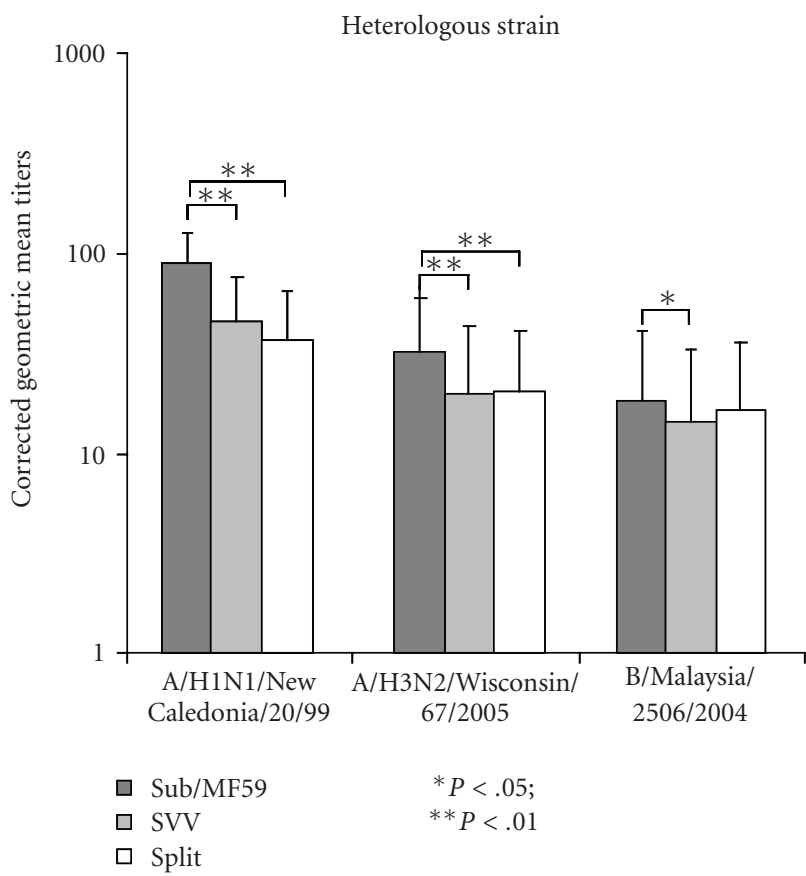

(b)

FIGURE 2: GMTs against homologous and heterologous influenza strains, according to vaccine group.

Significantly more subjects achieved at least a 4-fold increase in titres following Sub/MF59 vaccination against all three heterologous strains compared with SVV, and against both A drifted strains when compared with split group (Table 2). The rate of 4 -fold increase in HI titers versus B heterologous strain was significantly higher in the split group compared with SVV, and similar to the Sub/MF59 group.

As shown in Table 3, the multivariate analysis of variables associated with 4 -fold increase in $\mathrm{HI}$ antibody titers revealed that the presence of previous vaccination, only for the $\mathrm{B}$ strain, and the type of vaccine used, for all strain, were strong predictors of immune response against heterologous influenza strains.

\section{Discussion}

Vaccination is of crucial importance in preventing infection and protecting the vulnerable elderly population from disease but, over the past decade, a large number of studies have shown that antibody responses after vaccination are lower in elderly persons than in young adults, and that a variety of vaccines are less efficient in elderly persons [27].

Although the relationship between specific anti-influenza virus antibody levels and clinical protection is intrinsically variable [28], with other factors contributing to protection, such as antibodies to neuraminidase [29] and cellular immunity [30], antibody titers against hemaglutinin and derived surrogate end-points are currently considered the basis for licensure of influenza vaccines in the different age groups.
The use of an adjuvant to enhance the antibody response has been considered for a long time to be a valid option to increase the efficacy of influenza vaccines [31,32]. Preclinical studies have shown that the adjuvant MF59 is internalized by dendritic cells at the site of injection and facilitates internalization of the antigen to enhance the immune response and produce more antibodies.

This study was performed to evaluate the immunogenicity, using a validated $\mathrm{HI}$ assay, of three different inactivated influenza vaccines against both homologous and heterologous influenza strains in all evaluable sera from an original trial in nursing elderly residents with chronic underlying diseases. The original study population mainly comprised very elderly individuals, most of them with at least one underlying medical condition.

Although the sera were retested approximately 10 years after the original study, the data clearly confirmed the previous published evaluations vs. homologous antigens, showing higher immunogenicity in the adjuvanted vaccine group and the lower immune responses in the virosomal group [6]. After correction according to pre-vaccination status, a statistically significant difference in HI titers was evident in favor of MF59-adjuvanted vaccine against all three vaccine strains, when compared with the split vaccine, and against $\mathrm{A} / \mathrm{H} 3 \mathrm{~N} 2$ and $\mathrm{B}$ antigens when compared with the split vaccine. Although all three inactivated vaccines induced very high seroprotection rates, 4 -fold increase in titers was consistently higher in the MF59-adjuvanted vaccine group.

In addition, the MF59-adjuvanted influenza vaccine induced a higher level of immunogenicity against strains 


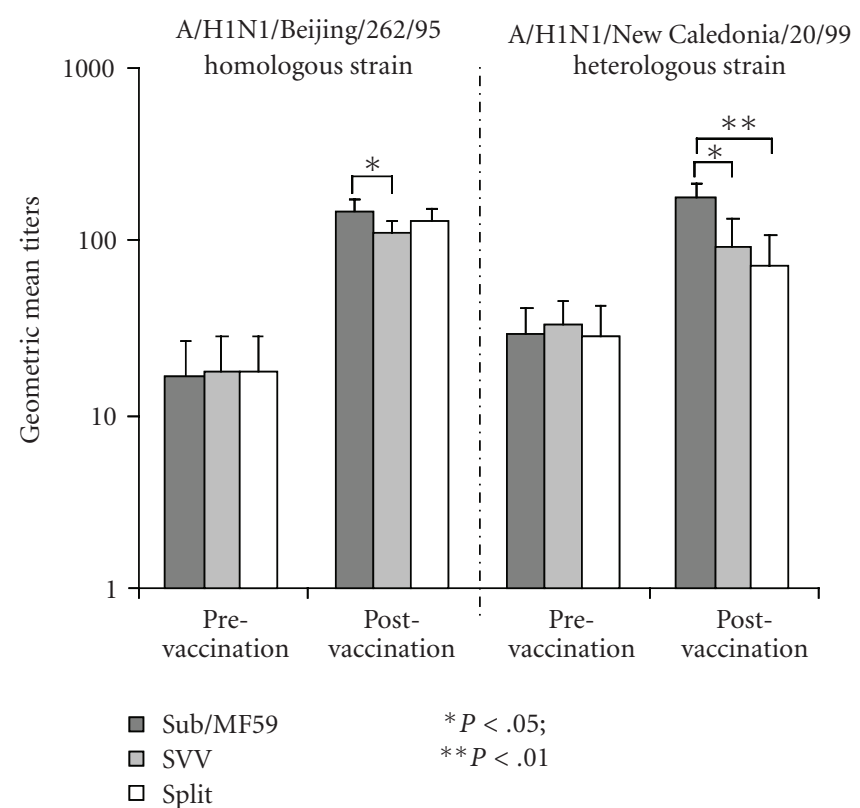

(a)

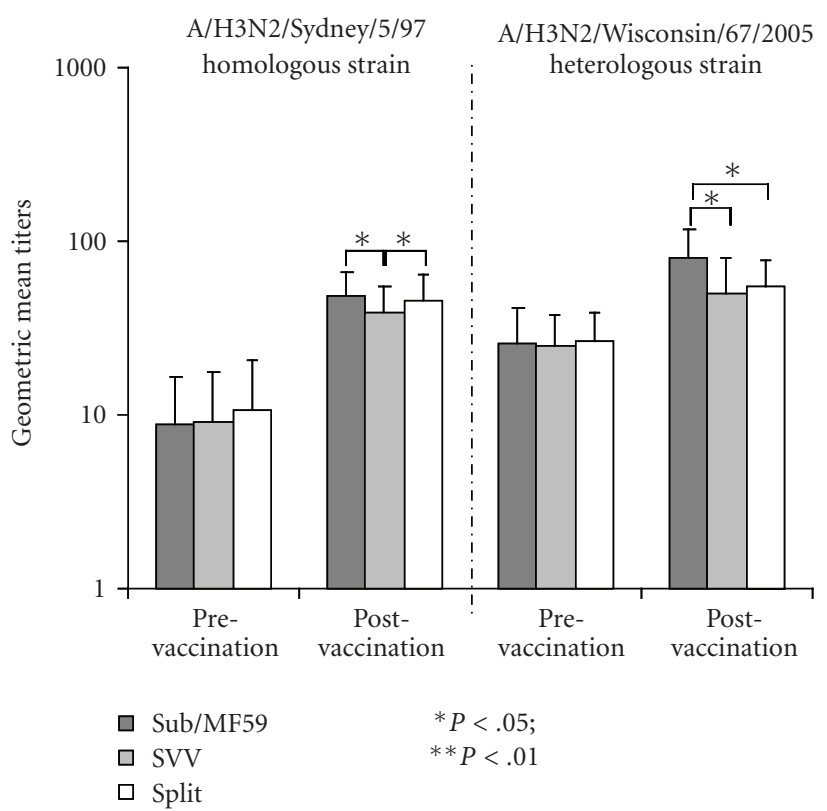

(b)

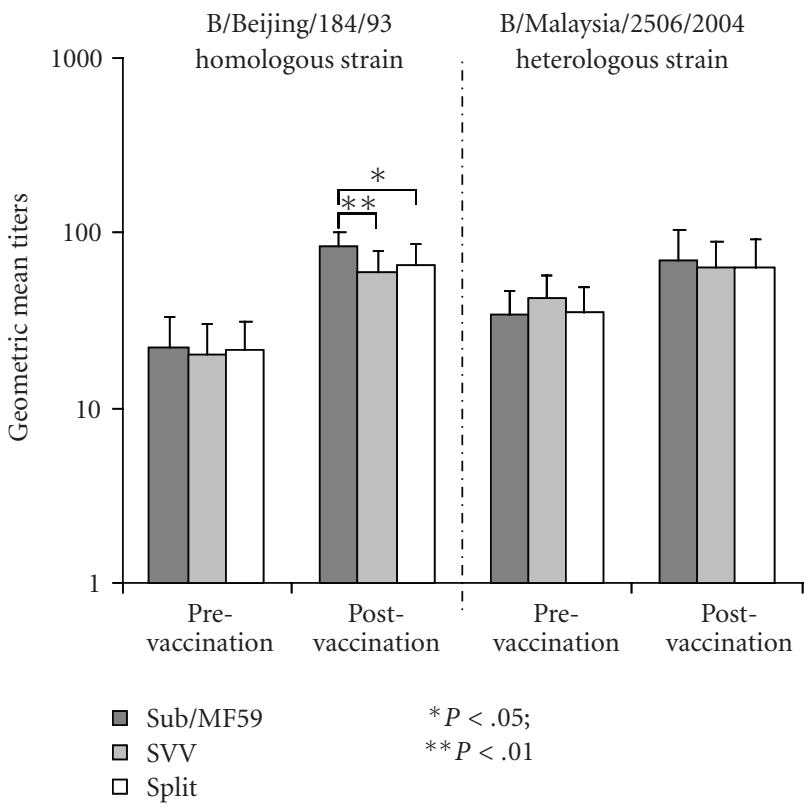

(c)

FIGURE 3: Corrected GMTs against homologous and heterologous influenza strains, according to vaccine group.

that bear no close antigenic similarity to those included in the vaccine formulation. For both heterologous A influenza strains, Sub/MF59 induced significantly higher HI antibodies and 4-fold increases in titers than the two non-adjuvanted comparators. For the B drifted strain, significantly higher HI titers were induced by MF59 adjuvanted vaccine, compared with SVV, and both adjuvanted and conventional split vaccine showed the highest increases in titers, compared with the virosomal vaccine.
Multivariate analysis also revealed that the presence of previous vaccination and the type of vaccine used were strong predictors of immune response against heterologous influenza strains (Table 3).

All vaccines fulfilled all three CHMP criteria for elderly against homologous strains. Against A heterologous antigens, only Sub/MF59 fulfilled all CHMP criteria versus $\mathrm{A} / \mathrm{H} 3 \mathrm{~N} 2$ and $\mathrm{A} / \mathrm{H} 1 \mathrm{~N} 1$ strains, SVV met all criteria against $\mathrm{A} / \mathrm{H} 1 \mathrm{~N} 1$ and none of them for $\mathrm{A} / \mathrm{H} 3 \mathrm{~N} 2$, Split vaccine 
TABLE 2: Mean Fold Increase in titres (MFI), Seroprotection $(\geq 40)$, and 4-fold increase rates according to viral strain and vaccine group.

\begin{tabular}{|c|c|c|c|c|}
\hline \multirow{2}{*}{ Viral strain } & \multirow{2}{*}{ Vaccine } & \multirow[t]{2}{*}{ MFI } & \multicolumn{2}{|c|}{ After vaccination (\%) } \\
\hline & & & Titre $\geq 40$ & 4-fold \\
\hline \multicolumn{5}{|c|}{$\mathrm{A} / \mathrm{H} 1 \mathrm{~N} 1-$ like virus } \\
\hline \multicolumn{5}{|l|}{ A/Beijing/262/95 } \\
\hline \multirow[t]{3}{*}{ (Homologous) } & Sub/MF59 & 8.7 & 100 & 94.4 \\
\hline & SVV & 6.3 & 100 & 84.6 \\
\hline & Split & 7.7 & 98.9 & 85.2 \\
\hline \multicolumn{5}{|c|}{ A/New Caledonia/20/99 } \\
\hline \multirow[t]{3}{*}{ (Heterologous) } & Sub/MF59 & 6.1 & 87.57 & $68.17 * * 7$ \\
\hline & SVV & 2.9 & $82.1 \quad *$ & $43.6^{-1 *} \mid *$ \\
\hline & Split & 2.4 & $68.2^{\perp}$ & 31.8 \\
\hline \multicolumn{5}{|c|}{$\mathrm{A} / \mathrm{H} 3 \mathrm{~N} 2$-like virus } \\
\hline \multicolumn{5}{|c|}{ A/Sydney/5/97 } \\
\hline \multirow[t]{3}{*}{ (Homologous) } & Sub/MF59 & 5.5 & 77.8 & 76.4 \\
\hline & SVV & 4.2 & 74.4 & 61.5 \\
\hline & Split & 4.2 & 79.5 & 62.5 \\
\hline \multicolumn{5}{|c|}{ A/Wisconsin/67/2005 } \\
\hline \multirow[t]{3}{*}{ (Heterologous) } & Sub/MF59 & 3.1 & $79.27 * *$ & $41.77 * * 7$ \\
\hline & SVV & 2.0 & $56.4]_{* *}^{* *}$ & $22.9^{J^{2 *}} \mid * *$ \\
\hline & Split & 2.0 & $78.4^{\rfloor^{* *}}$ & 27.3 \\
\hline \multicolumn{5}{|l|}{ B-like virus } \\
\hline \multicolumn{5}{|l|}{ B/Beijing/184/93 } \\
\hline \multirow[t]{3}{*}{ (Homologous) } & Sub/MF59 & 3.8 & 100 & $66.77_{* *}$ \\
\hline & SVV & 2.9 & 97.4 & $46.2^{-}$ \\
\hline & Split & 3.3 & 97.7 & 54.5 \\
\hline \multicolumn{5}{|c|}{ B/Malaysia/2506/2004 } \\
\hline \multirow[t]{3}{*}{ (Heterologous) } & Sub/MF59 & 2.0 & 69.4 & $25.07_{* *}$ \\
\hline & SVV & 1.5 & 71.8 & $7.7 与_{* *}$ \\
\hline & Split & 1.9 & 73.9 & $26.1\rfloor^{* *}$ \\
\hline
\end{tabular}

${ }^{*} P<.01 ; * * P<.05$.

TABLE 3: Multivariate analysis of variables associated with 4-fold HI-antibody titer increases according to heterologous virus strains.

\begin{tabular}{lcccccc}
\hline Variables & \multicolumn{2}{c}{ A/H1N1/NewCaledonia/20/99 } & \multicolumn{2}{c}{ A/H3N2/Wisconsin/67/2005 } & \multicolumn{2}{c}{ B/Malaysia/2506/2004 } \\
& OR & $95 \%$ CI & OR & 95\% CI & OR & 95\% CI \\
\hline Age $(<85)$ & 0.784 & $(0.421-1.458)$ & 1.515 & $(0.829-2.768)$ & 0.681 & $(0.344-1.347)$ \\
Gender (males) & 0.465 & $(0.177-1.219)$ & 1.280 & $(0.507-3.234)$ & 1.239 & $(0.463-3.317)$ \\
Underlying diseases & 1.431 & $(0.783-2.615)$ & 0.897 & $(0.490-1.644)$ & 1.598 & $(0.811-3.149)$ \\
Previous vaccinations & 1.447 & $(0.738-2.835)$ & 0.806 & $(0.417-1.559)$ & 0.307 & $(0.151-0.626)$ \\
Prevaccination titre $(\geq 1: 40)$ & 1.803 & $(0.976-3.333)$ & 0.676 & $(0.355-1.286)$ & 0.891 & $(0.447-1.776)$ \\
Split versus Sub/MF59 & 0.287 & $(0.150-0.547)$ & 0.436 & $(0.226-0.842)$ & 0.795 & $(0.404-1.564)$ \\
SVV versus Sub/MF59 & 0.400 & $(0.180-0.893)$ & 0.402 & $(0.167-0.965)$ & 0.215 & $(0.060-0.774)$ \\
\hline
\end{tabular}

fulfilled only one criterion against both antigens. Against $\mathrm{B}$ drifted strain only one criterion was met (seroprotection rate) by all vaccines tested.

In conclusion, data from these immunogenicity analyses confirmed that MF59-adjuvanted influenza vaccine can offer greater and broader immunogenicity against influenza in elderly and very elderly individuals who are at high risk of influenza-related complications.

Studies to further evaluate the relationship between vaccines-induced immunogenicity against homologous and heterologous strains and clinical protection are clearly warranted. 


\section{Acknowledgment}

The study described in this paper was supported by a University Grant (MURST ex-60\%). The first two authors contributed equally to this work.

\section{References}

[1] W. W. Thompson, D. K. Shay, E. Weintraub, et al., "Mortality associated with influenza and respiratory syncytial virus in the United States," Journal of the American Medical Association, vol. 289, no. 2, pp. 179-186, 2003.

[2] L. Ferrucci, J. M. Guralnik, M. Pahor, M. C. Corti, and R. J. Havlik, "Hospital diagnoses, Medicare charges, and nursing home admissions in the year when older persons become severely disabled," Journal of the American Medical Association, vol. 277, no. 9, pp. 728-734, 1997.

[3] J. E. McElhaney, "The unmet need in the elderly: designing new influenza vaccines for older adults," Vaccine, vol. 23, supplement 1, pp. S10-S25, 2005.

[4] Ch. Van Hoecke, V. Prikazsky, I. Uto, and C. Menschikowski, "Immunogenicity of an inactivated split influenza vaccine in institutionalized elderly patients," Gerontology, vol. 42, no. 4, pp. 190-198, 1996.

[5] M. J. W. Sprenger, P. G. H. Mulder, W. E. P. Beyer, R. Van Strik, and N. Masurel, "Impact of influenza on mortality in relation to age and underlying disease, 1967-1989," International Journal of Epidemiology, vol. 22, no. 2, pp. 334-340, 1993.

[6] V. Baldo, T. Menegon, C. Bonello, A. Floreani, and R. Trivello, "Comparison of three different influenza vaccines in institutionalised elderly," Vaccine, vol. 19, no. 25-26, pp. 34723475, 2001.

[7] T. A. Reichert, L. Simonsen, A. Sharma, S. A. Pardo, D. S. Fedson, and M. A. Miller, "Influenza and the winter increase in mortality in the United States, 1959-1999," American Journal of Epidemiology, vol. 160, no. 5, pp. 492-502, 2004.

[8] W. W. Thompson, D. K. Shay, E. Weintraub, et al., "Influenzaassociated hospitalizations in the United States," Journal of the American Medical Association, vol. 292, no. 11, pp. 1333-1340, 2004.

[9] WHO 2003: Fact sheet no. 211., July 2009, http://www.who .int/mediacentre/factsheets/fs211/en/Web.

[10] K. L. Nichol, "The efficacy, effectiveness and cost-effectiveness of inactivated influenza virus vaccines," Vaccine, vol. 21, no. 16, pp. 1769-1775, 2003.

[11] D. Herndler-Brandstetter, S. Schwaiger, E. Veel, et al., "CD25expressing CD8 ${ }^{+} \mathrm{T}$ cells are potent memory cells in old age," Journal of Immunology, vol. 175, no. 3, pp. 1566-1574, 2005.

[12] L. Lazuardi, B. Jenewein, A. M. Wolf, G. Pfister, A. Tzankov, and B. Grubeck-Loebenstein, "Age-related loss of naive T cells and dysregulation of T-cell/B-cell interactions in human lymph nodes," Immunology, vol. 114, no. 1, pp. 37-43, 2005.

[13] B. Weinberger, D. Herndler-Brandstetter, A. Schwanninger, D. Weiskopf, and B. Grubeck-Loebenstein, "Biology of immune responses to vaccines in elderly persons," Clinical Infectious Diseases, vol. 46, no. 7, pp. 1078-1084, 2008.

[14] N. J. Cox and K. Subbarao, "Global epidemiology of influenza: past and present," Annual Review of Medicine, vol. 51, pp. 407$421,2000$.

[15] A. S. Monto, "Epidemiology and virology of influenza illness," American Journal of Managed Care, vol. 6, no. 5, pp. S255S264, 2000.
[16] J. Nordin, J. Mullooly, S. Poblete, et al., "Influenza vaccine effectiveness in preventing hospitalizations and deaths in persons 65 years or older in Minnesota, New York, and Oregon: data from 3 health plans," Journal of Infectious Diseases, vol. 184, no. 6, pp. 665-670, 2001.

[17] J. Treanor, "Influenza caccine-outmaneuvering antigenic shift and drift," New England Journal of Medicine, vol. 350, no. 3, pp. 218-220, 2004.

[18] J. C. de Jong, W. E. P. Beyer, A. M. Palache, G. F. Rimmelzwaan, and A. D. M. E. Osterhaus, "Mismatch between the 1997/1998 influenza vaccine and the major epidemic $\mathrm{A}(\mathrm{H} 3 \mathrm{~N} 2)$ virus strain as the cause of an inadequate vaccine-induced antibody response to this strain in the elderly," Journal of Medical Virology, vol. 61, no. 1, pp. 94-99, 2000.

[19] F. Ansaldi, S. Bacilieri, F. Banfi, et al., "Neutralizing and hemagglutination-inhibiting activities of antibodies elicited by the 2004-2005 influenza vaccine against drifted viruses," Clinical and Vaccine Immunology, vol. 13, no. 1, pp. 162-164, 2006.

[20] G. D. Giudice, A. K. Hilbert, R. Bugarini, et al., "An MF59-adjuvanted inactivated influenza vaccine containing A/Panama/1999 (H3N2) induced broader serological protection against heterovariant influenza virus strain A/Fujian/2002 than a subunit and a split influenza vaccine," Vaccine, vol. 24, no. 16, pp. 3063-3065, 2006.

[21] N. Kojimahara, A. Maeda, T. Kase, and N. Yamaguchi, "Crossreactivity of influenza A (H3N2) hemagglutination-inhibition antibodies induced by an inactivated influenza vaccine," Vaccine, vol. 24, no. 33-34, pp. 5966-5969, 2006.

[22] F. Ansaldi, S. Bacilieri, P. Durando, et al., "Cross-protection by MF59-adjuvanted influenza vaccine: neutralizing and haemagglutination-inhibiting antibody activity against A(H3N2) drifted influenza viruses," Vaccine, vol. 26, no. 12, pp. 1525-1529, 2008.

[23] T. Menegon, V. Baldo, C. Bonello, D. Dalla Costa, A. Di Tommaso, and R. Trivello, "Influenza vaccines: antibody responses to split virus and MF59-adjuvanted subunit virus in an adult population," European Journal of Epidemiology, vol. 15, no. 6, pp. 573-576, 1999.

[24] Y. Kanegae, S. Sugita, A. Endo, et al., "Evolutionary pattern of the hemagglutinin gene of influenza B viruses isolated in Japan: cocirculating lineages in the same epidemic season," Journal of Virology, vol. 64, no. 6, pp. 2860-2865, 1990.

[25] K. Tamura, J. Dudley, M. Nei, and S. Kumar, "MEGA4: molecular evolutionary genetics analysis (MEGA) software version 4.0," Molecular Biology and Evolution, vol. 24, no. 8, pp. 1596-1599, 2007.

[26] W. E. P. Beyer, A. M. Palache, G. Luchters, J. Nauta, and A. D. M. E. Osterhaus, "Seroprotection rate, mean fold increase, seroconversion rate: which parameter adequately expresses seroresponse to influenza vaccination?" Virus Research, vol. 103, no. 1-2, pp. 125-132, 2004.

[27] T. R. Cate, "Clinical manifestations and consequences of influenza," American Journal of Medicine, vol. 82, no. 6, pp. 15-19, 1987.

[28] J. J. P. Nauta, W. E. P. Beyer, and A. D. M. E. Osterhaus, “On the relationship between mean antibody level, seroprotection and clinical protection from influenza," Biologicals, vol. 37, no. 4, pp. 216-221, 2009.

[29] A. S. Monto and A. P. Kendal, "Effect of neuraminidase antibody on Hong Kong influenza," The Lancet, vol. 1, no. 7804, pp. 623-625, 1973. 
[30] B. D. Forrest, M. W. Pride, A. J. Dunning, et al., "Correlation of cellular immune responses with protection against cultureconfirmed influenza virus in young children," Clinical and Vaccine Immunology, vol. 15, no. 7, pp. 1042-1053, 2008.

[31] E. D. Kilbourne, "Inactivated influenza vaccines," in Vaccines, A. Plotkin and E. A. Mortimer, Eds., pp. 565-581, Saunders, Philadelphia, Pa, USA, 1994.

[32] M. L. Clements and I. Stephens, "New and improved vaccines against influenza," in New Generation Vaccines, pp. 545-570, Marcel Dekker, New York, NY, USA, 2nd edition, 1997. 


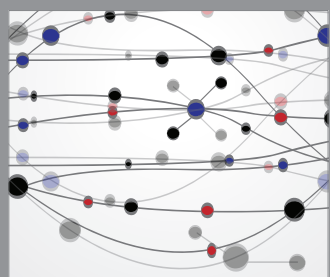

The Scientific World Journal
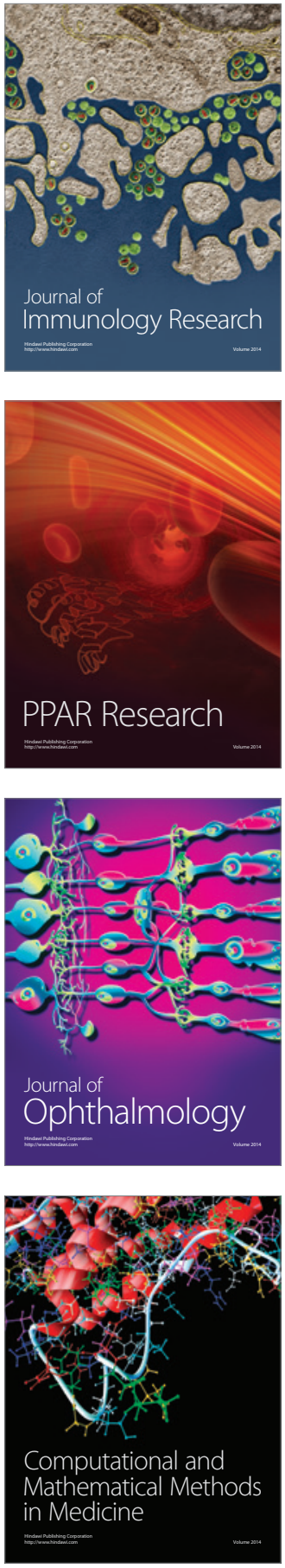

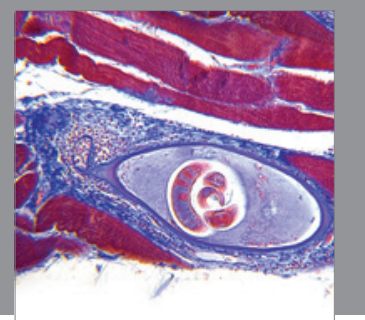

Gastroenterology

Research and Practice
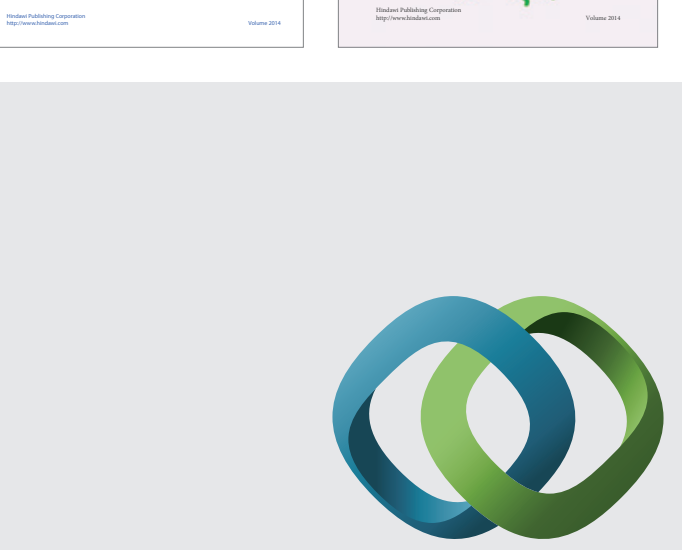

\section{Hindawi}

Submit your manuscripts at

http://www.hindawi.com
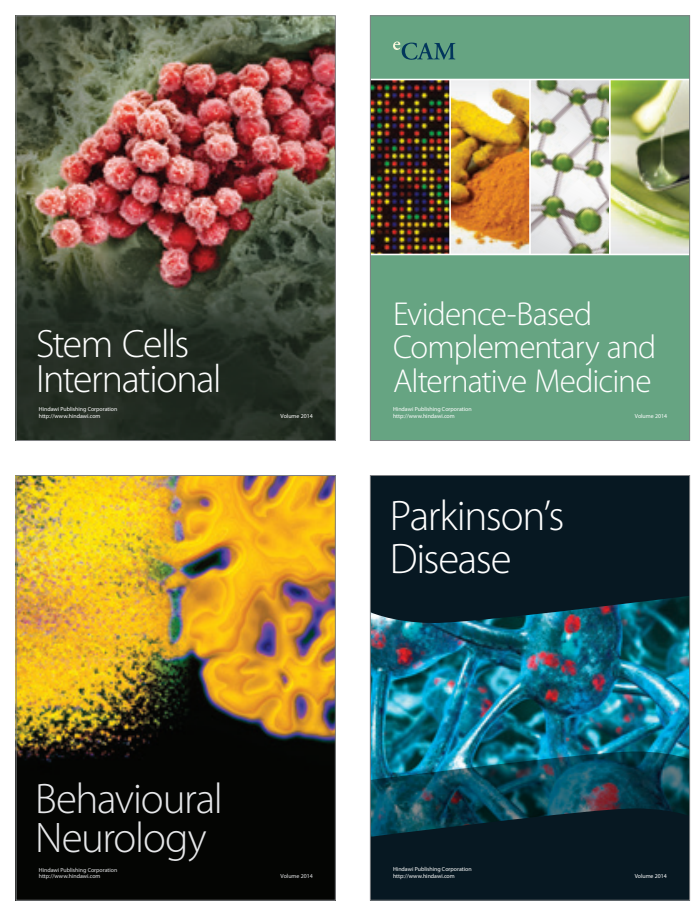

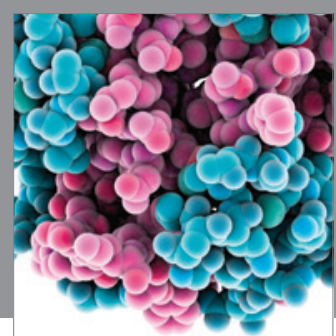

Journal of
Diabetes Research

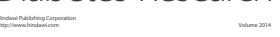

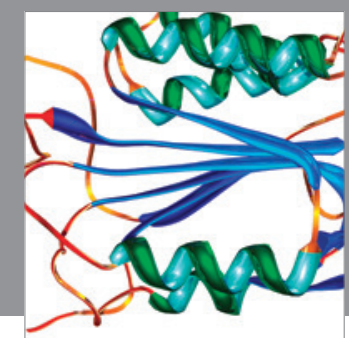

Disease Markers
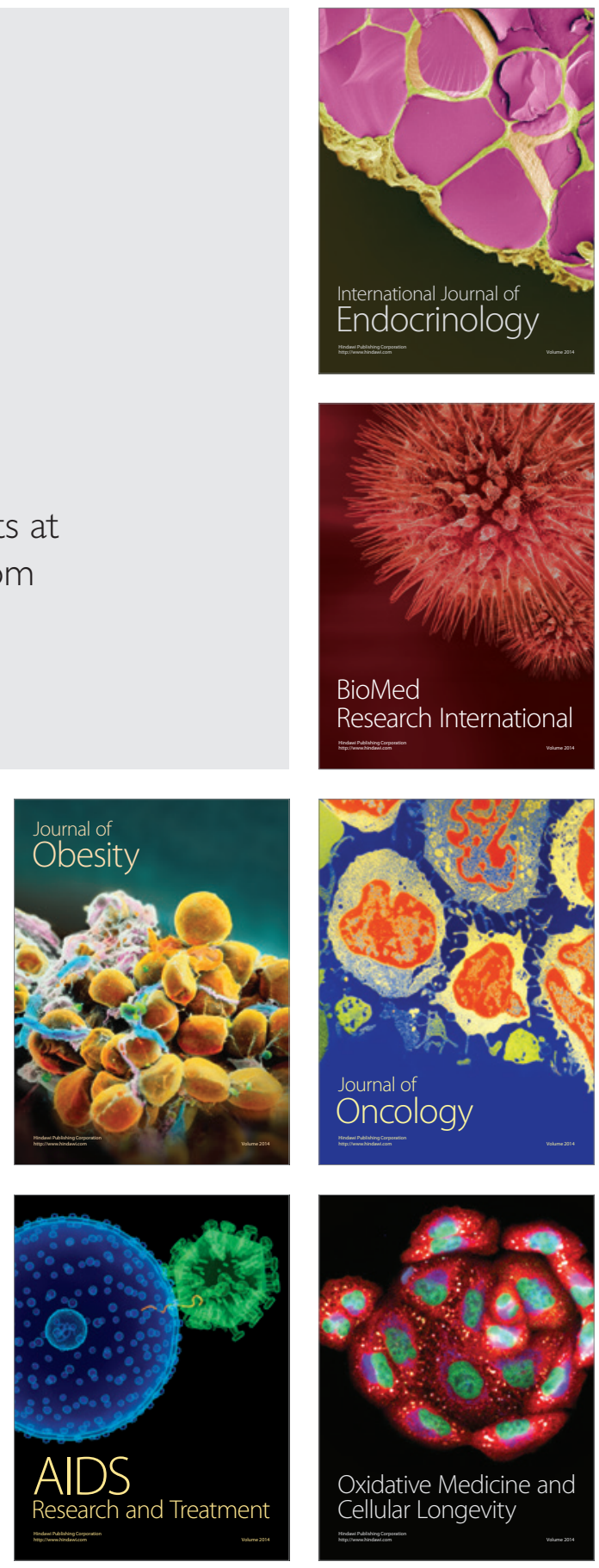